\title{
Randomized Comparison of Bipolar versus Unipolar Plus Bipolar Recordings During Segmental Ostial Ablation of Pulmonary Veins
}

\author{
HIROSHI TADA, M.D., HAKAN ORAL, M.D., BRADLEY P. KNIGHT, M.D., \\ MEHMET OZAYDIN, M.D., AMAN CHUGH, M.D., CHRISTOPH SCHARF, M.D., \\ SOHAIL HASSAN, M.D., RADMIRA GREENSTEIN, M.D., FRANK PELOSI, JR., M.D., \\ S. ADAM STRICKBERGER, M.D., and FRED MORADY, M.D.
}

From the Division of Cardiology, Department of Internal Medicine, University of Michigan, Ann Arbor, Michigan

\begin{abstract}
Unipolar vs Bipolar Electrograms. Introduction: Segmental ostial ablation to isolate pulmonary veins is guided by pulmonary vein potentials. The aim of this prospective randomized study was to compare the utility of unipolar plus bipolar electrograms versus only bipolar electrograms as a guide for segmental ablation to isolate the pulmonary veins in patients with atrial fibrillation.

Methods and Results: Isolation of the left superior, right superior, and left inferior pulmonary veins was attempted in 44 patients (35 men and 9 women; mean age $54 \pm 10$ years) with paroxysmal atrial fibrillation. A decapolar Lasso catheter was positioned in the pulmonary veins, near the ostium, and a conventional ablation catheter was used for segmental ablation aimed at elimination of all pulmonary vein potentials. One hundred fourteen pulmonary veins were randomly assigned for ostial ablation guided by either bipolar or unipolar plus bipolar recordings. Electrical isolation was achieved in $51(96 \%)$ of 53 pulmonary veins randomized to the bipolar approach, and $57(93 \%)$ of 61 pulmonary veins randomized to the unipolar plus bipolar approach $(P=0.7)$. In the unipolar plus bipolar group, the total duration of radiofrequency energy needed to achieve isolation, $5.5 \pm 2.8$ minutes/vein, was significant shorter than in the bipolar group, $7.6 \pm 4.1 \mathrm{minutes} / \mathrm{vein}(\mathrm{P}<0.01)$. Mean procedure and fluoroscopy durations per vein were $19 \%$ to $28 \%$ shorter in the unipolar plus bipolar group.

Conclusion: Segmental ostial ablation to isolate the pulmonary veins can be achieved more efficiently and with less radiofrequency energy when guided by both unipolar and bipolar recordings than by bipolar recordings alone. (J Cardiovasc Electrophysiol, Vol. 13, pp. 851-856, September 2002)
\end{abstract}

atrial fibrillation, pulmonary vein, unipolar electrogram

\section{Introduction}

Segmental ablation to isolate the pulmonary veins is guided by pulmonary vein potentials recorded near the ostia of the pulmonary veins..$^{1-5}$ By post hoc analysis, a recent study indicated that unipolar electrograms may provide better discrimination between successful and unsuccessful ostial ablation sites than bipolar electrograms. ${ }^{6}$ The aim of this prospective randomized study was to compare the utility of unipolar and bipolar electrograms as a guide for segmental ablation to isolate the pulmonary veins in patients with atrial fibrillation.

\section{Methods}

\section{Patient Characteristics}

The subjects of this study were 44 patients with drugrefractory, paroxysmal atrial fibrillation who underwent segmental ostial ablation to isolate the pulmonary veins.

Supported in part by a grant from the Gunma Prefecture Government and the Ellen and Robert Thompson Atrial Fibrillation Research Fund.

Address for correspondence: Fred Morady, M.D., Division of Cardiology, Department of Internal Medicine, University of Michigan, 1500 E. Medical Center Drive, B1F245, Ann Arbor, MI 48109-0022. Fax: 734-936-7026; E-mail: fmorady@umich.edu

Manuscript received 5 April 2002; Accepted for publication 3 July 2002.
There were 35 men and 9 women (mean age $54 \pm 10$ years). Mean duration of symptomatic atrial fibrillation was $8.5 \pm$ 7.8 years, and mean number of symptomatic episodes per month was $13 \pm 11$. One patient had coronary artery disease, and the remaining 43 patients had no structural heart disease. Echocardiography demonstrated a mean left ventricular ejection fraction of $0.62 \pm 0.09$ and a mean left atrial diameter of $39 \pm 4 \mathrm{~mm}$.

\section{Study Protocol}

The study protocol was approved by the Institutional Review Board. Therapy with antiarrhythmic drugs was discontinued at least 48 hours before the procedure. In the case of amiodarone, therapy was discontinued at least 6 weeks before the procedure. After obtaining informed consent, a quadripolar electrode catheter was inserted into a femoral vein and positioned in the coronary sinus. Transseptal catheterization was performed and selective pulmonary venous angiograms were performed to identify the location of the ostia. A 7-French decapolar ring catheter with 1-mm electrodes spaced at intervals of 4.5 or $6.0 \mathrm{~mm}$ (Lasso $^{\mathrm{TM}}$; Biosense Webster, Diamond Bar, CA, USA) and a 7-French quadripolar ablation catheter with a 4-mm distal electrode, an embedded thermistor, interelectrode spacing of 2-5-2 $\mathrm{mm}$, and a deflectable tip (EP Technologies, San Jose, CA, USA) were inserted into the left atrium. Heparin was administered as needed to maintain an activated clotting time 
of 250 to 350 seconds. ECG leads and intracardiac electrograms were recorded digitally (EPMed Systems, Mount Arlington, NJ, USA). Pacing was performed with a stimulator (model EP-3 Clinical Stimulator; EPMed Systems) at twice diastolic threshold and pulse width of $2 \mathrm{msec}$.

Isolation of the left superior, left inferior, and right superior pulmonary veins was attempted in all patients. ${ }^{5}$ Fifteen pulmonary veins were excluded from randomization because of incessant atrial fibrillation that precluded ablation during sinus rhythm or coronary sinus pacing. In two patients, the left superior and inferior pulmonary veins had a common ostium; for the purpose of analysis, these two veins were categorized as left superior pulmonary veins. Data from the right inferior pulmonary vein were not included in this study because the Lasso catheter usually could not be positioned within this vein and because isolation of this vein was attempted in only nine patients.

\section{Randomization}

Each targeted pulmonary vein was randomly assigned to ablation guided by either bipolar or unipolar plus bipolar recordings. When a pulmonary vein was randomized to bipolar recordings, no unipolar electrograms were displayed to the operators. When a pulmonary vein was randomized to unipolar recordings, unipolar electrograms served as the primary guide for ablation, but because a prior study demonstrated that small pulmonary vein potentials may not be detected by unipolar recordings, ${ }^{6}$ bipolar electrograms also were available for review. In the pulmonary veins randomized to the unipolar/bipolar approach, only $4 \%$ of ostial ablation sites were selected based on analysis of bipolar recordings.

The Lasso catheter was positioned inside the target pulmonary vein, within $5 \mathrm{~mm}$ of the ostium. Bipolar electrograms were recorded simultaneously with 10 pairs of adjacent electrodes of the Lasso catheter and with the distal pair of electrodes of the ablation catheter. The filter settings for the bipolar recordings were 30 to $500 \mathrm{~Hz}$. An indifferent electrode on the right thigh was used for unipolar recordings. Unipolar electrograms were recorded with each of the 10 electrodes of the Lasso catheter and with the distal electrode of the ablation catheter. The filter settings for the unipolar recordings were 0.05 to $200 \mathrm{~Hz}$. The bipolar and unipolar electrograms were recorded at a gain of $0.4 \mathrm{mV} /$ $\mathrm{cm}$.

\section{Selection of Ostial Ablation Sites}

Catheter ablation was performed during sinus rhythm or coronary sinus pacing at a cycle length of $600 \mathrm{msec}$, depending on the technique that provided the best separation of pulmonary vein and atrial potentials. ${ }^{7,8}$ If overlap between the atrial and pulmonary vein potentials was present during both sinus rhythm and coronary sinus pacing, a single atrial extrastimulus was introduced to separate the potentials. ${ }^{9}$

In the bipolar electrogram group, the only parameter used to identify ablation sites was the relative timing of pulmonary vein potentials. The Lasso catheter bipoles that demonstrated the earliest pulmonary vein potentials were identified, and the ablation catheter was positioned on the ostial side of the Lasso catheter, adjacent to these bipoles, where there was a pulmonary vein potential that was simul- taneous with or earlier than the earliest pulmonary vein potential recorded by the Lasso catheter (Fig. 1).

In the unipolar electrogram group, the primary parameters used to select ablation sites were the steepness and amplitude of the intrinsic deflection, and a secondary parameter was its relative timing. The use of these parameters was based on the results of a prior study. ${ }^{6}$ The Lasso catheter electrode that recorded the steepest and largest intrinsic deflection was identified, and the ablation catheter was positioned adjacent to this electrode (Fig. 1). Before delivery of radiofrequency energy, correct positioning of the ablation catheter was verified by identical or very similar morphologies of the unipolar electrograms recorded by the ablation catheter and the target Lasso catheter electrode (Fig. 1). ${ }^{6}$

\section{Radiofrequency Ablation}

To minimize the risk of pulmonary vein stenosis, radiofrequency ablation was performed using a maximum power of 30 to $35 \mathrm{~W}$ and a maximum electrode-tissue interface temperature of $52^{\circ} \mathrm{C} .5$ The applications of energy were 30 to 60 seconds in duration. The endpoint of ablation was elimination of all ostial pulmonary vein potentials and complete

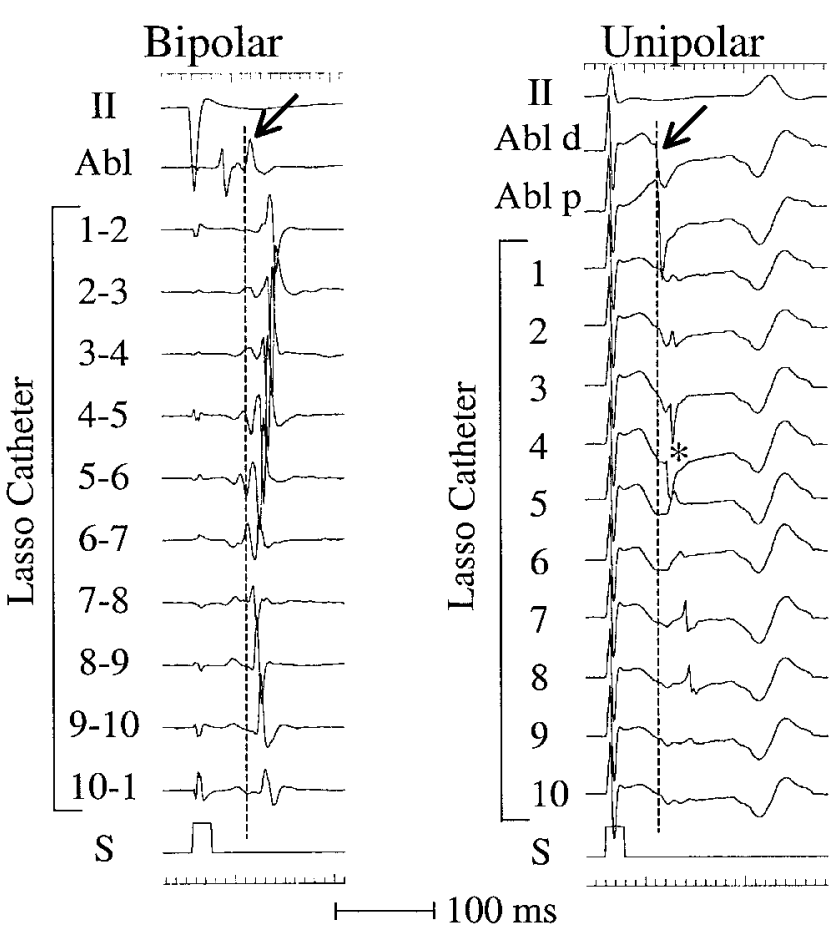

Figure 1. Examples of criteria used to select ostial ablation sites using bipolar and unipolar recordings. In both examples, the recordings were performed during coronary sinus pacing at a cycle length of $600 \mathrm{~ms}$. In the bipolar recordings, the pulmonary vein potential (arrow) recorded by the ablation catheter preceded the earliest pulmonary vein potential recorded by the Lasso catheter. The vertical dashed line marks the onset of the bipolar pulmonary vein potential recorded by the ablation catheter. In the unipolar recordings, the steepest and largest intrinsic deflection recorded by the Lasso catheter was at electrode 4 (asterisk), and the distal electrode of the ablation catheter was positioned near this electrode, such that the pulmonary vein potentials recorded by the ablation catheter (arrow) and electrode 4 were very similar. The vertical dashed line marks the onset of the unipolar pulmonary vein potential recorded by the ablation catheter. $A b l=$ ablation catheter, $d=$ distal, $p=$ proximal; $S=$ stimulus. 
Figure 2. Example of endpoints for ostial ablation. (A) Elimination of all pulmonary vein potentials (arrows) recorded in a left superior pulmonary vein during coronary sinus pacing. (B) Complete entrance block into a left inferior pulmonary vein during induced atrial fibrillation. $A b l=$ ablation catheter; $C S=$ coronary sinus, $d=$ distal, $p=$ proximal; $L A=$ left atrium; $S=$ stimulus.
A.

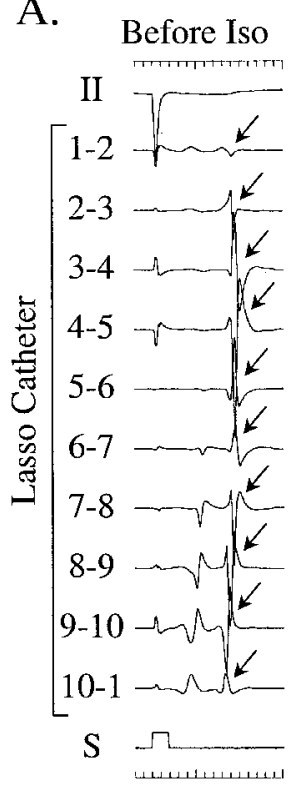

After Iso

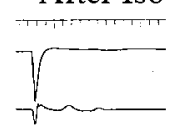

$\rightarrow+$
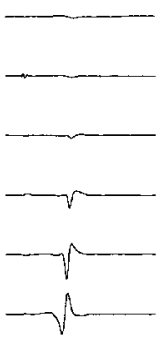

$\sim r$
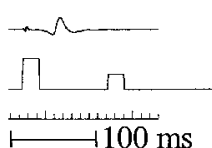

B.

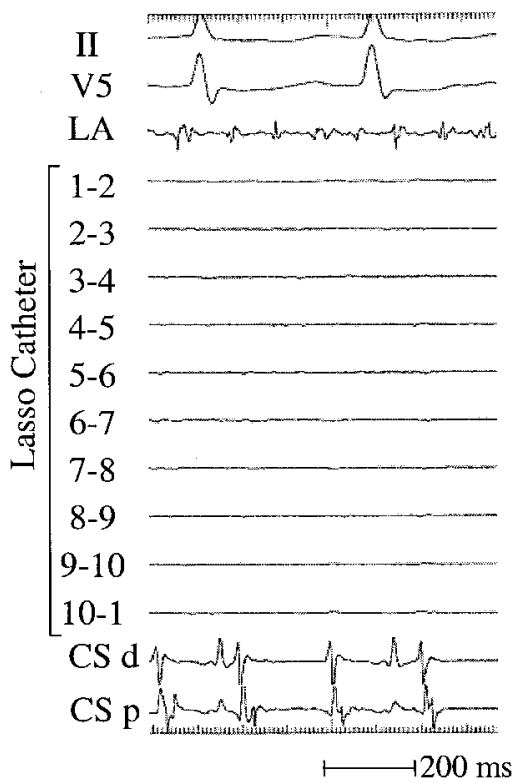

entrance block into the pulmonary vein during pacinginduced atrial fibrillation (Fig. 2).

\section{Follow-Up}

The patients were hospitalized for 24 hours after the ablation procedure, underwent continuous telemetric monitoring, and were treated with intravenous heparin. Upon discharge from the hospital, the patients were treated with low-molecular-weight heparin for 4 to 5 days and with warfarin for 1 to 3 months.

The patients were seen in an outpatient clinic 4 weeks after the procedure and every 3 to 4 months thereafter. All patients were asked to contact a study nurse in the event of recurrent symptoms. All patients who reported symptoms were given an event monitor to document the cause of symptoms. Computed tomography was performed 3 months after the ablation procedure to look for pulmonary vein stenosis. The follow-up interval was $>3$ months in all patients.

\section{Study Endpoints}

Five endpoints were compared between the bipolar and unipolar groups: (1) successful electrical isolation; (2) amount of time needed to achieve isolation, starting at the time the Lasso catheter was positioned within the vein and ending when there was complete electrical isolation; (3) duration of fluoroscopy per vein; (4) total duration of radiofrequency energy applications per vein; and (5) pulmonary vein stenosis.

\section{Statistical Analysis}

Continuous variables are expressed as mean $\pm 1 \mathrm{SD}$ and were compared using Student's $t$-test. Dichotomous variables were compared by Chi-square analysis. $\mathrm{P}<0.05$ was considered statistically significant.

\section{Results}

\section{Randomization}

One hundred fourteen pulmonary veins were eligible for randomization. Fifty-three pulmonary veins were randomized to the bipolar group, and 61 were randomized to the unipolar/bipolar group. There were no significant differences in the locations of the pulmonary veins between the two groups $(\mathrm{P}=0.9$, Table 1$)$.

\section{Successful Isolation}

Complete electrical isolation was achieved in 51 (96\%) of the 53 pulmonary veins randomized to the bipolar approach, and 57 (93\%) of the 61 pulmonary veins randomized to the unipolar/bipolar approach $(\mathrm{P}=0.7)$. The percentage of the ostial circumference that was ablated to achieve electrical isolation was $59 \% \pm 20 \%$ in the bipolar group and $55 \% \pm 22 \%$ in the unipolar/bipolar group ( $\mathrm{P}=$ $0.5)$.

\section{Other Study Endpoints}

Procedure duration, fluoroscopy time, and total duration of radiofrequency energy applications all were significantly shorter in the unipolar/bipolar group than in the bipolar group (Table 2). Mean procedure duration per vein was 19\% shorter in the unipolar/bipolar group; mean fluoroscopy

TABLE 1

Locations of Pulmonary Veins in the Bipolar and Unipolar/Bipolar Groups

\begin{tabular}{lcc}
\hline & Bipolar & Unipolar/Bipolar \\
\hline Total number & 53 & 61 \\
Left superior & $19(36 \%)$ & $19(31 \%)$ \\
Right superior & $18(34 \%)$ & $23(38 \%)$ \\
Left inferior & $16(30 \%)$ & $19(31 \%)$ \\
\hline
\end{tabular}


TABLE 2

Procedural Endpoints: Comparison Between Bipolar and Unipolar/Bipolar Groups

\begin{tabular}{llll}
\hline & Bipolar & Unipolar/Bipolar & P Value \\
\hline $\begin{array}{l}\text { Procedure duration } \\
\quad \text { min/vein) }\end{array}$ & $33.6 \pm 16.8$ & $27.3 \pm 14.3$ & $<0.05$ \\
$\begin{array}{l}\text { Fluoroscopy time (min/vein) } \\
\text { Total duration of RF energy }\end{array}$ & $15.5 \pm 9.5$ & $11.2 \pm 6.7$ & $<0.01$ \\
$\quad$ application (min/vein) & $7.6 \pm 4.1$ & $5.5 \pm 2.8$ & $<0.01$ \\
\hline
\end{tabular}

Values expressed as mean $\pm 1 \mathrm{SD}$.

$\mathrm{RF}=$ radiofrequency .

time per vein was $28 \%$ shorter; and mean duration of radiofrequency energy applications was $28 \%$ shorter.

\section{Follow-Up}

During mean follow-up period of $225 \pm 56$ days, atrial fibrillation recurred in $14(32 \%)$ of the 44 patients. The other 30 patients $(68 \%)$ were free from recurrent episodes of atrial fibrillation and were not treated with any antiarrhythmic drugs.

Three of the 14 patients with recurrent atrial fibrillation underwent a second ablation procedure. In 2 of the 3 patients, all three pulmonary veins had been isolated using unipolar plus bipolar electrograms during the first ablation session. One of these patients had recovery of conduction in all three pulmonary veins, and the other had recovery of conduction in the left superior and right superior pulmonary veins. In the remaining patient, the left superior and inferior pulmonary veins had been isolated using bipolar electrograms, and the right superior pulmonary vein had been isolated using unipolar plus bipolar electrograms. In this patient, there was recovery of conduction in all three pulmonary veins.

\section{Pulmonary Vein Stenosis}

There was no evidence of pulmonary vein stenosis in any of the 53 pulmonary veins in which ablation was guided by bipolar electrograms or in any of the 62 pulmonary veins in which ablation was guided by unipolar plus bipolar electrograms.

\section{Discussion}

\section{Major Findings}

The results of this study demonstrate that segmental ostial ablation to isolate pulmonary veins can be performed more efficiently when guided by a combination of unipolar and bipolar recordings than when guided by bipolar recordings alone. The acute success rate for isolation of the pulmonary veins was approximately $95 \%$ in this study, regardless of whether bipolar or unipolar recordings were used to guide ablation. However, unipolar electrograms recorded at the ostia of the pulmonary veins and by the ablation catheter allowed for more precise targeting of pulmonary vein potentials. This resulted in a significant reduction in the amounts of time, fluoroscopy, and radiofrequency energy needed to achieve complete isolation.

\section{Advantages of Unipolar Over Bipolar Ostial Recordings}

The possible explanations for the superiority of unipolar recordings over bipolar recordings when attempting to identify ostial ablation sites to eliminate pulmonary vein potentials were described in a prior study. ${ }^{6}$ First, unipolar recordings of pulmonary vein potentials provide more information useful for localization than bipolar recordings. The steepness and amplitude of the intrinsic deflection in unipolar recordings are related to the proximity of the recording electrode to the site of origin of a depolarization. ${ }^{10}$ Because of the complex arrangement of muscle fascicles in the muscle sleeves that surround the pulmonary veins, ${ }^{11}$ the information provided by the intrinsic deflection of the unipolar electrogram allows more precise localization than bipolar electrograms, which provide only the relative timing of the pulmonary vein potentials (Fig. 3).

Second, when pulmonary vein potentials are recorded in bipolar fashion with a Lasso catheter, the particular electrode that is closest to the source of the pulmonary vein potential may not be clear (Fig. 3). Similarly, when a pulmonary vein potential is recorded with an ablation cath-
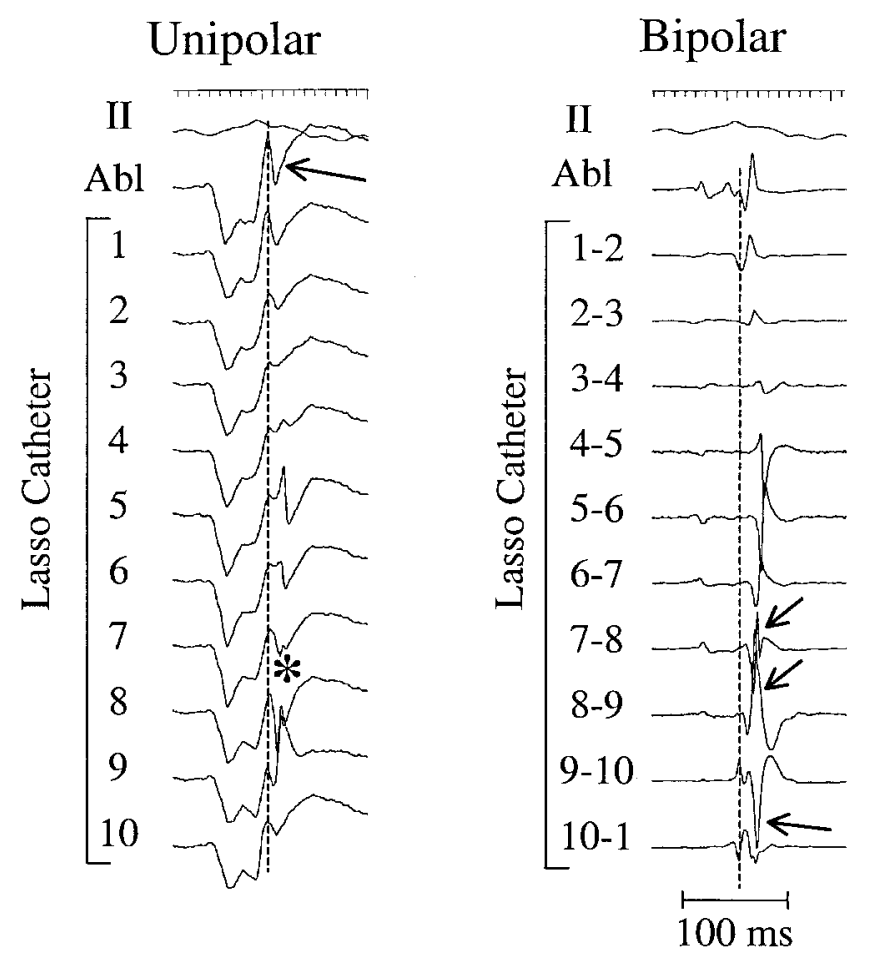

Figure 3. Example of a successful ostial ablation site identified by analysis of unipolar recordings from the right superior pulmonary vein during sinus rhythm. A steep intrinsic deflection was recorded at electrode 8 of the Lasso catheter (asterisk), and the ablation catheter was maneuvered to an adjacent site where the unipolar electrograms recorded by the ablation catheter (arrow) and by electrode 8 were similar. The vertical dashed line marks the onset of the unipolar pulmonary vein potential recorded by the ablation catheter. Also shown are the bipolar electrograms recorded at the same site and analyzed post hoc. Note that the bipolar pulmonary vein potentials recorded by electrodes 7-8, 8-9, and 9-10 (arrows) all appear to have the same timing and that the appropriate electrode at which to position the ablation catheter would not have been clear based on analysis of these electrograms. The vertical dashed line marks the onset of the bipolar pulmonary vein potential recorded by the ablation catheter. Abl= ablation catheter. 

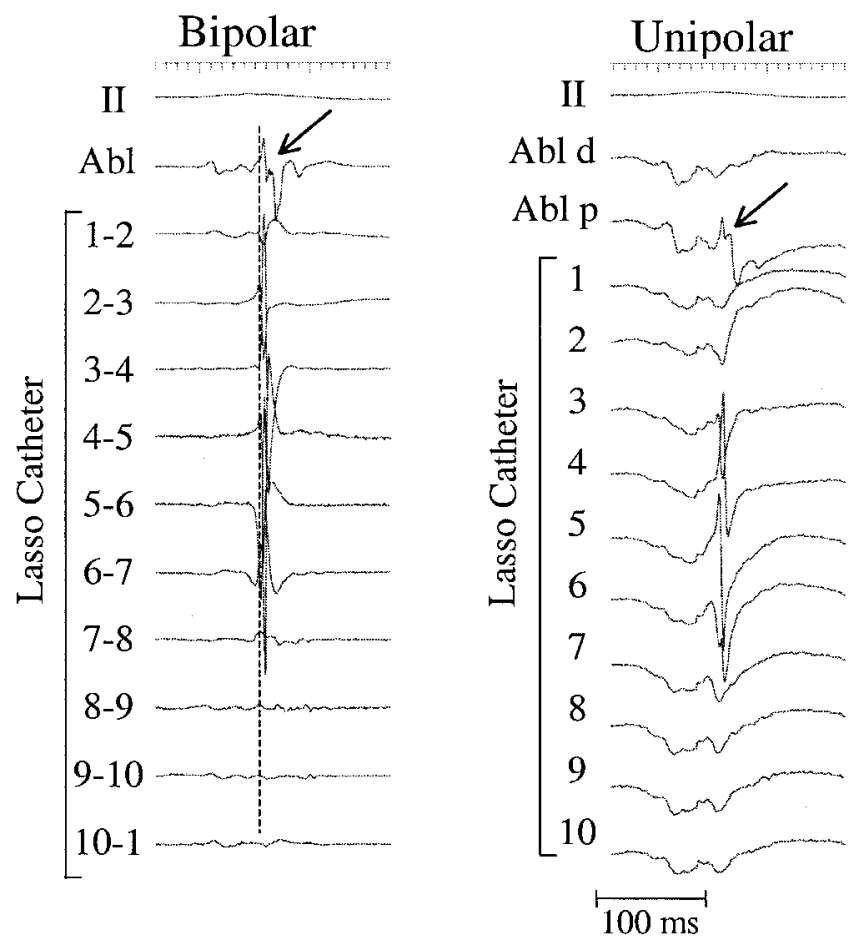

Figure 4. Example of an unsuccessful ostial ablation site selected based on analysis of bipolar recordings from the right superior pulmonary vein during sinus rhythm. A two-component pulmonary vein potential was recorded by the ablation catheter, but an application of radiofrequency energy at this site had no effect. The vertical dashed line marks the onset of the bipolar pulmonary vein potential recorded by the ablation catheter. Post hoc analysis of the unipolar electrograms recorded at the same site demonstrated that the pulmonary vein potentials were recorded from the proximal electrode of the ablation catheter distal bipole. If this pulmonary vein had been randomized to the unipolar approach, the unsuccessful application of radiofrequency energy at this site would have been avoided. Abbreviations as in Figure 1.

eter, it may be the anode that is closest to the source of the potential, not the distal electrode through which radiofrequency energy is delivered (Fig. 4). These sources of imprecision in localization of pulmonary vein potentials are eliminated when using unipolar recordings.

A third possible explanation for the superiority of unipolar recordings over bipolar recordings when attempting to identify ostial ablation sites to eliminate pulmonary vein potentials involves localization of the ablation catheter relative to the electrodes of the Lasso catheter. Because of the different orientation of the bipoles, the electrograms recorded by the ablation catheter are never identical to the Lasso catheter recordings. Therefore, visual cues provided by fluoroscopy or by more sophisticated localization tools are needed to guide positioning of the ablation catheter to the appropriate ostial ablation site, and the response to an application of radiofrequency energy is the only way to confirm that the ablation catheter was correctly positioned. In contrast, the morphology of the unipolar electrograms recorded with the ablation and Lasso catheters allows accurate localization of the ablation catheter relative to the electrodes of the Lasso catheter. This provides a technique for confirmation of correct positioning of the ablation catheter that is independent of fluoroscopy and that may avoid the delivery of ineffective applications of radiofrequency energy.

In the present study, it is likely that all three of these explanations accounted for the smaller amount of radiofrequency energy needed to achieve isolation of pulmonary veins when using unipolar recordings as opposed to bipolar recordings. However, the extent to which each possible advantage of unipolar recordings over bipolar recordings contributed to the greater efficiency of the ablation procedure was not determined.

At times, the amplitude of pulmonary vein potentials may be too small for detection by unipolar recordings. Therefore, bipolar recordings sometimes are necessary when using primarily unipolar electrograms to guide segmental ostial ablation. However, in the present study, only $4 \%$ of target sites in the unipolar/bipolar group were selected based on analysis of bipolar electrograms.

\section{Pulmonary Vein Stenosis}

Because the delivery of radiofrequency energy within pulmonary veins is avoided during segmental ostial ablation, this technique for electrical isolation of the pulmonary veins has not been associated with pulmonary vein stenosis when the power of radiofrequency energy applications is limited to 30 to $35 \mathrm{~W} .{ }^{1,5}$ Accordingly, there were no instances of pulmonary vein stenosis in the present study, regardless of whether the procedure was guided by unipolar or bipolar electrograms.

\section{Study Limitations}

A limitation of this study is that the long-term success rates of pulmonary vein isolation guided by bipolar and unipolar recordings are not available. Because randomization was based on individual pulmonary veins, not patients, both the bipolar and unipolar/bipolar approaches often were used in the same patient. Therefore, when atrial fibrillation recurred during follow-up, the particular pulmonary vein that was responsible for the recurrence was not known, except in the small number of patients who underwent a repeat ablation procedure.

Another limitation of the study is that, because a repeat ablation procedure was performed in only a small number of patients, the possibility that an extrapulmonary vein source was responsible for the atrial fibrillation usually could not be ruled out.

\section{Conclusion}

It is clear that excellent results can be achieved when segmental ostial ablation to isolate the pulmonary veins is guided by conventional bipolar recordings. ${ }^{1-4}$ However, any procedural refinement that results in a similar clinical outcome in a shorter period of time and requires less fluoroscopy time and radiofrequency energy is worthwhile. The present study demonstrates that the use of unipolar recordings provides this type of refinement.

\section{References}

1. Haissaguerre M, Jais P, Shah DC, et al: Electrophysiological end point for catheter ablation of atrial fibrillation initiated from multiple pulmonary venous foci. Circulation 2000;101:1409-1417.

2. Haissaguerre M, Shah DC, Jais P, et al: Electrophysiological break- 
throughs from the left atrium to the pulmonary veins. Circulation 2000;102:2463-2465

3. Shah DC, Haissaguerre M, Jais P, et al: Electrophysiologically guided ablation of the pulmonary veins for the curative treatment of atrial fibrillation. Ann Med 2000;32:408-416.

4. Shah DC, Haissaguerre M, Jais P, et al: Curative catheter ablation of paroxysmal atrial fibrillation in 200 patients: Strategy for presentations ranging from sustained atrial fibrillation to no arrhythmias. Pacing Clin Electrophysiol 2001;24:1541-1558.

5. Oral H, Knight BP, Tada H, et al: Pulmonary vein isolation for paroxysmal and persistent atrial fibrillation. Circulation 2002;105: 1077-1081.

6. Tada H, Oral H, Wasmer $\mathrm{K}$, et al: Pulmonary vein isolation: Comparison of bipolar and unipolar electrograms at successful and unsuccessful ostial ablation sites. J Cardiovasc Electrophysiol 2002;13:13-19.
7. Hocini M, Shah DC, Jais P, et al: Concealed left pulmonary vein potentials unmasked by left atrial stimulation. Pacing Clin Electrophysiol 2000;23:1832-1835.

8. Tada H, Oral H, Greenstein R, et al: Differentiation of atrial and pulmonary vein potentials recorded circumferentially within pulmonary veins. J Cardiovasc Electrophysiol 2002;13:118-123.

9. Tada H, Oral H, Ozaydin M, et al: Response of pulmonary vein potentials to premature stimulation. J Cardiovasc Electrophysiol 2002; 13:33-37.

10. de Bakker J, Hauer R, Simmers T: Activation mapping: Unipolar versus bipolar recording. In Zipes D, Jalife J, eds: Cardiac Electrophysiology: From Cell to Bedside. Second Edition. WB Saunders, Philadelphia, 1995.

11. Saito T, Waki K, Becker A: Left atrial myocardial extension onto pulmonary veins in humans: Anatomic observations relevant for atrial arrhythmias. J Cardiovasc Electrophysiol 2000;11:888-894. 